
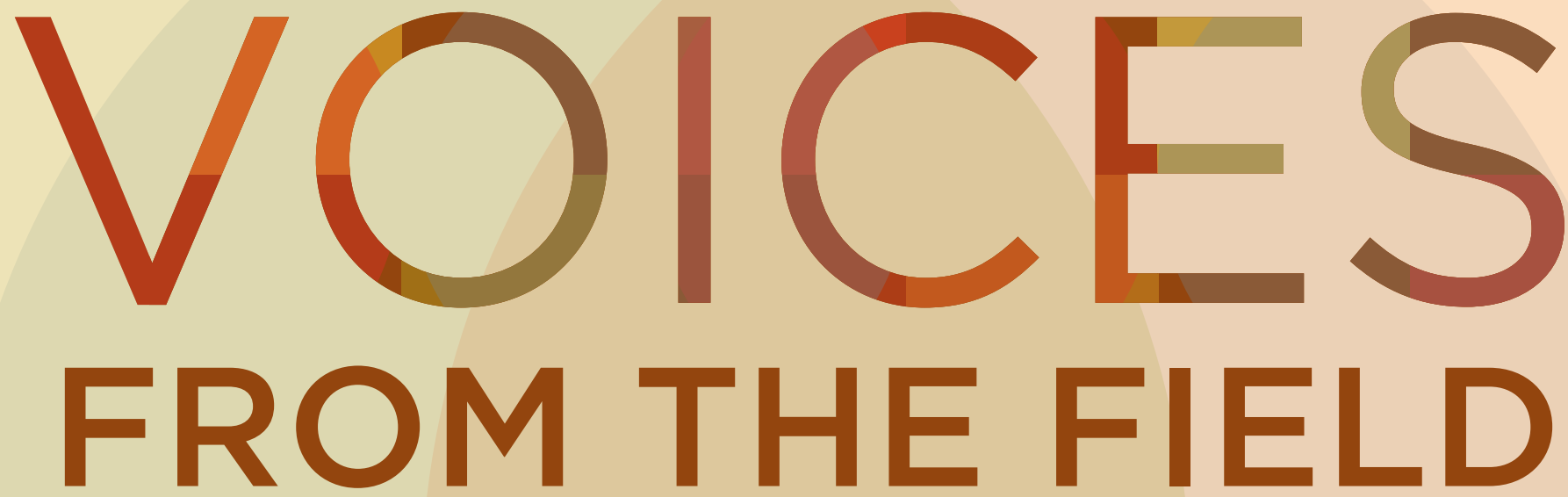

\title{
The Impact of COVID-19 on
}

Early Career Scholars and Doctoral Students

Felice J. Levine | Na'ilah Suad Nasir | Cecilia Rios-Aguilar Ryan Evely Gildersleeve | Katherine J. Rosich Megan Bang | Nathan E. Bell | Matthew A. Holsapple

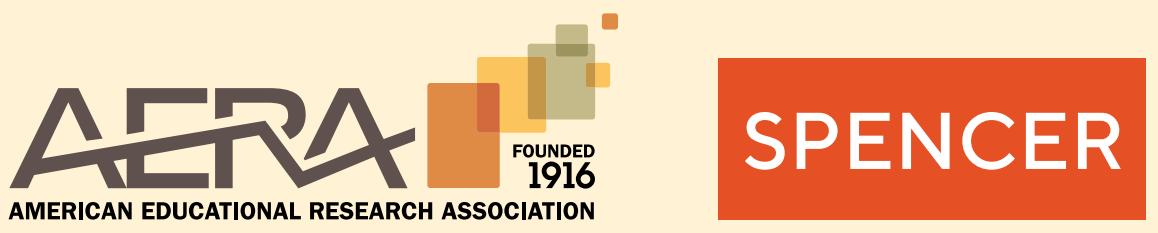


(c) American Educational Research Association and the Spencer Foundation

This report is a joint publication of the American Educational Research Association and the Spencer Foundation. The views, findings, and conclusions are those of the authors and do not necessarily reflect the views, positions, or policies of the American Educational Research Association or the Spencer Foundation.

The American Educational Research Association is the largest national interdisciplinary research association devoted to the scientific study of education and learning. Founded in 1916, AERA advances knowledge about education, encourages scholarly inquiry related to education, and promotes the use of research to improve education and serve the public good.

The Spencer Foundation has been a leading funder of education research since 1971. Spencer believes education research is integral to improving education, making education systems more equitable, and increasing opportunities to learn across the lifespan.

https://doi.org/10.3102/aera20211

\section{Preferred Citation:}

Levine, F. J., Nasir, N. S., Rios-Aguilar, C., Gildersleeve, R. E., Rosich, K. J., Bang, M., Bell, N. E., \& Holsapple, M. A. (2021). Voices from the field: The impact of COVID-19 on early career scholars and doctoral students [Focus group study report]. American Educational Research Association; Spencer Foundation. https://doi.org/10.3102/aera20211

Design: Kathleen Dyson 


\section{Contents}

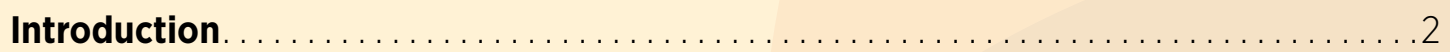

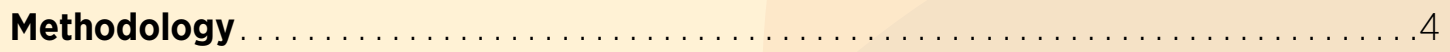

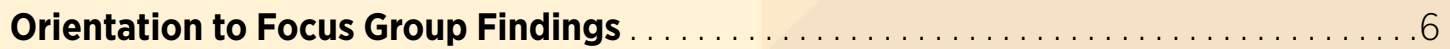

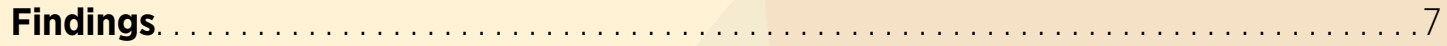

(1) Research Impact: Disruptions, Delays, and Adaptations . . . . . . . . . . . . . . . 8

(2) Impact on Teaching: The Need to Be Inventive, Inclusive, and Intentional . . . . . . 13

3 Balancing Acts: Negotiating Family, Home, Community, and Professional Life ... 15

(4) The Emergence of a Dual Pandemic and Confronting Racism ................ 18

5 Employment Trajectories, Uncertainties, and Deferments ................ 20

6 Institutional (In)Capacity to Respond and Support ....................... 23

(7) Emerging and Lost Connections, Communities, and Communication........... 26

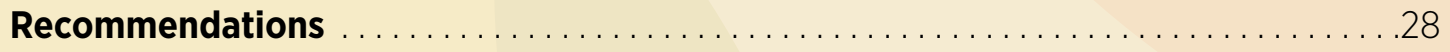

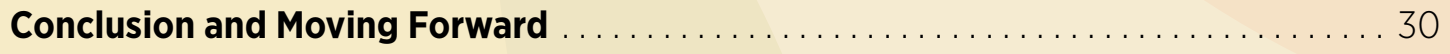

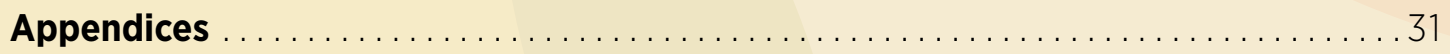

Appendix A: Focus Group Methodological Details . . . . . . . . . . . . . . . . . . . . . . 31

Appendix B: Focus Group Protocol. . . . . . . . . . . . . . . . . . . . . . . . . . . . 36

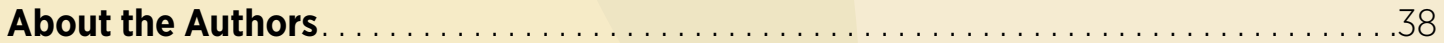




\section{Introduction}

n the spring of 2020, the COVID-19 public health crisis shook the world. In the United States in

March, in just a matter of days, businesses, restaurants, schools, and universities closed or took their functions online in a move of triage that was mirrored on a worldwide scale. Almost every sector was affected, and in higher education specifically, the impacts were large and deep. As universities and schools transitioned rapidly to online instruction, many faculty in schools of education were in the bind of needing to move their classes online, providing mentoring and reassurance to their students, handling enormous disruption to research projects as well as managing care for sick or elderly family members, caring for their children now in school via online instruction, and at the same time facing new professional responsibilities and challenges.

As scholars connected to two organizations in the field of education research, we were very much concerned with the well-being and career trajectories of education researchers. We knew that the pandemic could have a disproportionate impact on some groups, including early career faculty and researchers, women, scholars of color, and doctoral students. We wanted to lend our expertise and bandwidth in the moment and thought it would be an important contribution to listen to and gather information about the experiences of scholars and doctoral students at this critical time.

With these aims in mind, we embarked upon a joint inquiry of the American Educational Research Association (AERA) and the Spencer Foundation to explore how early career scholars ${ }^{1}$ and doctoral students coming into the field were being affected by changes in the wake of the pandemic. The value of this inquiry has only amplified in significance as the reach and duration of the pandemic continue, with consequences that are likely to be long and far ranging. Our

The original intent of the project was to undertake a survey to assess short-and long-term impacts of the COVID-19 pandemic. By our first full team meeting at the beginning of April 2020, we had decided to also conduct a series of focus groups to deepen our appreciation of the lived experiences of early career scholars and doctoral students as the pandemic was rapidly unfolding. aim is to provide information that colleges and universities, funders of research, and other organizations can use to better support the next generation of education researchers and help buffer or contain adverse impacts to them and to the field. ${ }^{2}$

The original intent of the project was to undertake a survey to assess short- and long-term impacts of the COVID-19 pandemic. By our first full team meeting at the beginning of April 2020, we had decided to also conduct a series of focus groups to deepen our appreciation of the lived experiences of early career scholars and doctoral students as the pandemic was rapidly unfolding. We were particularly interested in

1 For purposes of this study, early career scholars is defined as scholars who received their doctorates from 2013 through 2019 . The term scholars applies to all early career participants. Although the types of positions they held and the nature of their work varied, most were in academic positions or in research positions in higher education or other work sectors (see Appendix A).

2 Although this study (the AERA-Spencer COVID-19 Focus Group Study) focuses on the field of education research, we also hope that this project will contribute to a broader understanding of early career professionals and doctoral students in other scientific and scholarly fields in areas where relevant research might not have been undertaken. 
the impact on research and research careers. With approximately $80 \%$ of those with doctoral degrees in education research working as faculty or otherwise employed in higher education institutions, ${ }^{3}$ the focus group study emphasized that sector, although we also included focus groups that drew from the many other settings in which education research professionals work.

This report presents the focus group findings and recommendations. During the weeks of May 18 through June 1, 2020, we conducted 12 focus groups that by design included separate groups for early career scholars of color, doctoral students of color, and also women of color. It was evident from the beginning that the COVID-19 crisis was highly racialized and having a disproportionate adverse impact on communities of color. We thought our design should reflect our concern about these dynamics and the fact that the pandemic could exacerbate preexisting conditions. We also wanted to learn about any deeper impacts being experienced by those historically marginalized in academia.

These deeper impacts became even more salient for the four focus groups held on June 2-3 at the end of our focus group study, when the nation erupted in racial and social unrest as people took to the streets to protest the brazen and inhumane killing of George Floyd by police. ${ }^{4}$ In the focus groups that occurred at a time when systemic racism was on display in such a heightened way, our conversations with participants ${ }^{5}$ were intellectually intense and deeply emotional, both about the national context and about the ways in which scholars of color, and Black scholars in particular, face systemic racism and anti-Blackness as a normal part of their personal and professional lives. This report thus sheds light on the lived experience of early career scholars and doctoral students of color in relation to this second pandemic.

3 See Table 5, "Trends in AERA Membership by Sector of Employment," in N. E. Bell \& F. J. Levine, 2016, AERA Membership: A Demographic Profile and Trends, p. 8. Unpublished report, available through the American Educational Research Association.

4 George Floyd was killed on May 25, 2020, in Minneapolis, Minnesota, with expressions of protest and pain becoming national and international by the weekend preceding our final four focus groups on June 2-3, 2020.

5 While the emphasis of the focus groups remained the COVID-19 pandemic, the focus group leaders opened each discussion in the June 2-3 focus groups by acknowledging and expressing concerns about systemic racism, providing the space and opportunity for participants to speak to this issue to the extent they wished. The findings from these discussions are addressed in this report and were also valuable in refining the design of the survey. 


\section{Methodology}

efore presenting the findings, we briefly overview the design of and methods used in the COVID-19 Focus Group Study. The detailed methodology and research protocol for the conduct of the focus groups are set forth in Appendices A and B, respectively. ${ }^{6}$

\section{Sample}

The study population for the focus groups was drawn from the 2018 and 2019 AERA membership database for persons with the designation of graduate student or early career scholar within 7 years of their doctorate. Doctoral students were invited to participate from among graduate students attending "very high research activity" universities and "high research activity" universities. Early career scholars were invited to participate from universities with "very high research activity," from other higher education institutions of all types, and from work sectors outside of academia.? (See Appendix A for detailed information on sampling and recruitment.)

\section{Study Design}

The design called for 12 focus groups in order to ensure a representation of doctoral students and early career scholars in terms of diverse background characteristics, institutions of training, and work settings. This strategy achieved the goal of compositional diversity. With a higher participation rate for persons of color and women, however, the obtained sample included a substantially larger proportion of persons of color and women than did the early career and graduate student populations from which they were drawn. ${ }^{8}$ Across the 12 groups, ${ }^{9}$ there were 58 participants, with 86\% (50) women and 69\% (40) persons of color. ${ }^{10}$

\section{Structure and Format of Focus Groups}

The focus group meetings (1.5 hours long each) were held through Zoom and were video recorded. The data derived directly from the participants in each group as they shared their views on and experiences with the COVID-19 pandemic. The focus group protocol had three components (see Appendix B): (1) brief welcome, reminder of the study's aims and human subjects' issues, and self-introductions; (2) an openended and fluid discussion of what was salient in the lives of early career scholars and doctoral students; and (3) a final discussion of what the research team should pay attention to in designing the survey.

\footnotetext{
6 The focus group study was reviewed and approved by the Institutional Review Board (IRB) at the American Institutes for Research that serves as the IRB fo research involving human participants undertaken by the American Educational Research Association.

7 The Carnegie Classification of Institutions of Higher Education was used to identify and place participants in the higher education focus groups. The Carnegie system classifies universities with "very high research activity" as R1 institutions and those with "high research activity" as R2 institutions. It also classifies other universities as Doctoral/Professional Universities. See Appendix A for the specification of the 12 focus groups and where the Carnegie classification system was used. The Carnegie system was updated in 2018 and is available at https://carnegieclassifications.iu.edu/classification_ descriptions/basic.php.

8 In the relevant study population overall, $71 \%$ and $72 \%$ of the early career scholars and graduate students, respectively, were women; $42 \%$ and $52 \%$ of the early career scholars and graduate students, respectively, were persons of color.

9 There were four focus groups comprised of persons of color, with overall 19 participants, 17 of whom were female. See Table 2, Appendix A

10 We were attentive to these compositional issues when we examined the focus group data and sought in reporting findings to capture the range of perspectives and perceptions rather than to draw conclusions about what was dominant or not. We see the focus groups as adding insights about the impact of COVID-19, despite our awareness of the limitations of the inferences we can draw.
} 
The second component of the protocol constituted the major part of the focus group. While guided by the general protocol, the format was open-ended from the outset, with participants being asked to introduce themselves and start with offering two words that captured their feelings about their situations at this COVID-19 time.

Topics arose organically; key issues included home-family-work balance and circumstances, current employment or work status, impacts on research, institutional messages and supports, financial situations and dislocations, research or other opportunities, effects on teaching, and so forth.

\section{Data Analysis}

The data analysis included a systematic study of the videos, transcriptions, and notes for all 12 focus groups to identify salient issues and themes. Also, the "two words" of focus group participants were examined as a way of anchoring on the mindset of research participants. Based on several analytic strategies, the research team identified salient themes that structured the findings presented in this report (for specific details on all aspects of the data analysis, see Appendix A). 


\section{Orientation to Focus Group Findings}

hroughout the focus group study and this report, our attention to the COVID-19 pandemic reflects
several key goals. We recognize that COVID-19 has created the conditions for higher education to change in unknown and unforeseen ways; this study captures just the beginning of that trajectory of change, although the beginning story is both important and telling. Like many times of inordinate challenge and rapid shifts, the current situation holds promise as well as peril. This could be a defining moment for our field to lean into abandoning the kinds of practices that may have outlived their usefulness and elevating those that can lead to greater equity, inclusivity, humanity, and respect for early career scholars and doctoral students.

We think it is critical, then, to learn from those with the most at stake in order to identify and provide opportunities for institutions and leaders to support this generation of researchers, who will have gone through an experience unprecedented in recent time.

We hope, by focusing on the pandemic during its rapid emergence and spread, to learn about what was being lost as well as what was becoming possible that may not have been possible before.

We thus offer this report humbly and as a beginning to a longer and more robust discussion once the survey is completed and the survey findings are fully analyzed. The findings and recommendations we present in this

We hope, by focusing on the pandemic during its rapid emergence and spread, to learn about what was being lost as well as what was becoming possible. report are not conclusive, but rather suggestive. We do not claim that what we are reporting is generalizable for all early career researchers, especially given the limited scope and number of our focus groups. But we do think there are important lessons to learn in how we might better support early career researchers and each other, as scholars of education. We view these lessons as being relevant for higher education leaders, senior faculty, funders of research, professional association leaders, and perhaps more. 


\section{Findings}

The findings are reported from the vantage of seven key themes that evolved empirically. Before we well-being in two words at the outset of the focus group sessions. Then, we detail each of the themes, leaning heavily on the voices of participants as we do so.

\section{Two Words to Start}

As part of the self-introductions, participants shared two words that captured their feelings. These feelings ranged significantly, reflecting the complexity of the moment and the participants' desire to manage multiple demands while still remaining as positive as possible. Some used words such as "anxious," "worried," or "stressed”; "chaotic," "unsettled," or "upheaval”; or "exhausted," "tired," and "challenged." Others used words that indicated their focus on gratitude for what they did have, and their adaptability, ranging from "calm" and "relaxed" to "fine" and "hopeful" to "thankful," "optimistic," or "better than ... expected." Words conveying emotions like "anger," "frustration," "rage," or "outrage" were also present, particularly at the time of the national protests in the wake of the murder of George Floyd in June. As a doctoral student of color put it on June 2, "I am feeling 'saddened' and 'sickened'” (FG10-23, very high research activity university)."1

Many participants were experiencing ambivalent emotions. They said they were experiencing "fears," "stress," and "uncertainties," and yet at the same time said they felt "relieved," "calm," "steady," "privileged," and "hopeful." Participants overall seemed to be trying, despite serious apprehensions, to adjust to their circumstances and their interest in moving ahead. An education researcher working for and teaching at her university observed:

And I guess my two words would be "overwhelmed," because there is a lot happening, it seems, all at once, and my inbox is ridiculous every morning, even more so than it was before. And also, I think maybe "steady," because, in a lot of ways, where things were in a lot of turmoil at the midpoint in the semester, they feel with the semester ending-it's usually very bittersweet for me when the semester ends and we have graduation, and this particular end of this semester, I'm just so happy it's over. And it was successful, and people are at various levels of okay, and the summer semester is starting. And there's some kind of steady comfort in that cycle of the semester. So maybe that's also hopeful in that, okay, things are moving forward in ways that we can expect, even when there's a lot of uncertainty. I FG01-32, VERY HIGH RESEARCH ACTIVITY UNIVERSITY, CLINICAL FACULTY

The opening words were salient as a place of departure for each of the focus group discussions. The findings from the thematic analyses provided more insight into these feelings, the struggles, the resilience, the worry, the stress, the anger, and the adaptations being made.

11 The quotes reference the academic affiliations of the focus group participants in the following ways: (1) doctoral students are referenced as at "very high research activity" or "high research activity" universities as set forth in the Carnegie Classification of Institutions of Higher Education, see footnote 7; (2) early career scholars are referenced as at "very high research activity" universities or at higher education institutions. Those employed in work sectors outside of academia are referenced in terms of the type of institutional employer. 


\section{(1) Research Impact: Disruptions, Delays, and Adaptations}

One key concern for participants was how their research projects and trajectories were being affected by the pandemic. The primary issue for the focus group study was the impact of COVID-19 on participants' research, how they were currently handling research projects, and what they thought they most needed, looking ahead. Even when research was underway, various levels of concern were expressed. Some participants emphasized derailments or delays; others noted changes they had made or were contemplating making. Less than three months after the onset of the pandemic, participants were reporting that their research lives and their studies had changed. Participants recognized that education across levels and contexts was also rapidly changing and thus research would undergo or need to undergo rapid changes as well.

An early career scholar captured this uncertainty, ambiguity, and attention to the future:

I think that many things, many of the things that we do [are] going to change drastically and systematically. So, what happens to a school? Are they going to be the same school as we know [it]? What happens to universities? And I feel like very huge shifts are going or coming our way, and we have no idea of what's going to happen and how to get ready for that, that, you know, we might think that, is it even good to ... do more research on how to teach science as ideal when we are not sure that there is going to be a science classroom anymore in near future....

So, there are things that are going to change so huge and drastically that we cannot even talk about, ... I think -I am the person who [is] just trying to ignore them because it's so huge that we cannot think about that. But they are there. ... So, there are so many questions that are there that we are not going to answer easily, but, you know, maybe we need some forums of actually imagining collectively about that. I FG04-48, VERY HIGH RESEARCH ACTIVITY UNIVERSITY, RESEARCHER

For some, the necessary changes required a complete overhaul of projects that were still in development or that relied on in-person fieldwork. These circumstances engendered concerns for career trajectories as research productivity was threatened by the closure of preK-12 schools, community organizations, and higher education institutions, and by out-of-school learning contexts. Whether education researchers were located in tenure-track, contingent faculty, or postdoctoral positions; in research organizations; or still in doctoral programs, the pandemic's possibility for upending research projects was real. Many education researchers were left wondering if their planned research projects would be seriously delayed or happen at all.

I actually had just solidified a future partnership with a middle school right as it happened. Like, we were having our final meeting the week that everything locked down and obviously, you know, I tried reaching out, but I also understand their whole focus is getting their kids online and getting their families online. . . I FG03-41, HIGHER EDUCATION INSTITUTION, FACULTY 
... So, then I'm feeling that, being in my second year, I feel like this is where l'm

supposed to be the most productive, where l'm supposed to have papers under review and being productive, so that by the time I'm up for tenure, I have the publications that I need. And so, the papers that are under review have taken over a year, are still under review, and so l'm feeling like I can't-l'm not-I don't have the [new] data to write right now. Everything is frozen. Everything is stopped. I FG02-35, VERY HIGH RESEARCH ACTIVITY UNIVERSITY, FACULTY

Delays were not localized to specific types of research, methods, or modes of inquiry. A doctoral student who was not collecting original data made a similar point. He was no longer able to have access to restricted data at his university, resulting in his dissertation being put on hold (FG06-12, very high research activity university, doctoral student).

Some participants reported making shifts and were matter-of-fact about it. Others expressed concerns about pivoting, including about possible inequities in new studies that focused on easier-to-reach populations:

I just try to remain optimistic, ... oh, my dissertation; that's the other thing l'm working on. That changed too. I was going to go into two schools, two early childcare centers to collect data, but now with the closure and everything kind of changing in those regards and us not knowing, the kind of uncertainty, I'm going to a more theoretical dissertation. So, in that front, while looking for a job and doing the whole moving thing, still working on that and working on getting that into kind of a more conceptual, theoretical type of study. So, yeah, just been trying to stay optimistic. ... I FG08-29, HIGH RESEARCH ACTIVITY UNIVERSITY, DOCTORAL STUDENT

But I've really had to ... rethink research projects that I had started. ... [W] we were doing family math workshops in the community, and l've worked since August to build relationships with families in the communities, and then suddenly, we can't do those workshops anymore.... So, we've had to shift to thinking about what ways can we understand families' math experiences, and we really have... limited ways of connecting with and understanding the experiences of communities of color, particularly from low-income backgrounds. ... So, the research that we've been able to do ... we recognize we're now doing research with more privileged populations. I FG04-49, VERY HIGH RESEARCH ACTIVITY UNIVERSITY, FACULTY

Focus group participants emphasized the impact of the pandemic not just on research but also on writing. Many commented that women and other caregivers had significant family responsibilities that might have short- and long-term career consequences. Some scholars reported that they had worked intensively to sustain or even increase their research productivity during the early months of the pandemic. Their reasons ranged from a commitment to their work, to a concern that any decrease could be detrimental to their career, to a sense that they ought to take advantage of the opportunity to get more done when institutions' shifting to remote work made it more possible. 
So, I am pumping out the research. All the stuff, all the stuff that I heard recently that men are actually submitting more right now than women-and I want-I want to change that, and so I just finished one article, and I-you know, that we've been working on for a while now, you know, and a couple of people are looking at it. And l'm now starting another one, and this is stuff that we've been working on for years but that [has] just been sitting there. And so, I'm finally getting it together. I FG05-53, HIGHER EDUCATION INSTITUTION, ADJUNCT

For others, even without caregiving responsibilities, sustained and exclusive attention to writing remained a challenge in the midst of COVID-19:

I understand I'm very fortunate. I don't have kids. I wasn't teaching, right? Like, in some ways, feeling like I shouldn't even be allowed to feel this, so much anxiety, right-but also, like, yeah, I might not be teaching. I might not have kids, but that doesn't mean I could write, and, like, I can't-you know, [my] being released from teaching when I was supposed to go out and do research, right? Like I can balance research and writing. I can balance teaching and writing. I can't write all day in the middle of a pandemic. I FG12-18, VERY HIGH RESEARCH ACTIVITY UNIVERSITY, POSTDOC

Some early career scholars emphasized the need for resources in their professional contexts to support them in planning for changes to their research due to the impact of the pandemic. And, for researchers working in academic institutions that were not "very high research activity," a strain on resources meant that research funding was even less available.

I'm pre-tenure. l'm going into my fifth year.... So, it's an extremely stressful, like, time and year, and there's definitely been a lot of focus on teaching and online teaching ... and a lot of support for that, ... I'm at a point now where I really need some extra research and scholarship support and attention because very little has been given to that ... I had data collection that was happening in schools, and that just suddenly came to a halt. And so, all the data we had collected before that now just has-I don't even know what to do with it, honestly, because I'm not even sure, research-wise, how I can make it into anything, because it was supposed to be a two-year data collection. I FG03-43, HIGHER EDUCATION INSTITUTION, FACULTY

Some also reported the absence of outreach from institutional or scholarly leadership to help them navigate their research activity and expectations given the challenges posed by the pandemic. Participants mentioned the importance of mentoring during the pandemic, both for their students and for themselves from more senior scholars. This interest in greater support included mentoring about how to manage and stay focused during the pandemic generally, but also how to conduct or adapt their research projects and stay productive. 
So, I wish there was more support right now with junior faculty, from senior faculty who can guide us as we're grappling with all these changes to our research, toward teaching. Everything is changing right now, and that overwhelming feeling is, I think-if we had a mentor who had already been through it and could guide us and hold our hands and, you know, ... just tell us it will be okay, like, "Are you okay? We'll get through this," I think that would make a difference with some guidance in our academic trajectory, if that makes sense. I FG02-35, VERY HIGH RESEARCH ACTIVITY UNIVERSITY, FACULTY

Participants observed as well that research productivity for many of them suffered from responding to institutional pulls and the need to do other kinds of work. The circumstances of the pandemic drew early career scholars' attention and expertise into various other forms of work, away from their research. For example, some were called upon to support local school districts in planning for remote learning; others were asked to pick up and prepare to teach extra courses at the last minute; and still others needed to place their attention on their home and family, as will be discussed further below. Participants recognized that their institutions were managing multiple parallel crises and appreciated the efforts of administrators and colleagues, but they also would have liked their institutions to provide more funds, more research support, and more senior-level guidance, as well as make expectations clearer, especially for pre-tenured faculty.

Those early in their careers working in sectors outside of academia reflected on similar complexities regarding the shift to working professionally from home and the impact of COVID-19 on their professional research roles. Their discussions identified two primary concerns. First, they emphasized the direct impact of COVID-19 on their everyday work, whether they were employed by schools or school systems or by research organizations responsible for research-related work. They were experiencing a huge challenge being unable to enter into classrooms and schools to continue doing their research.

Well, I would say as someone who develops curriculum, a huge pivot [is] to figure out how to do it differently, but without the access to the resources to do the type of research to make sure that it works, right? So, under typical circumstances, you know, you would pilot or field test a program in schools with kids, and now it's "Okay. Let's figure out how to do this distance learning" but without being able to rigorously test it and make sure that we're doing well, and so it's kind of like putting stuff out there and hoping that it works... I FGO9-10, RESEARCH ORGANIZATION, RESEARCHER

Second, they were keenly aware at a personal level that in order to continue on a career as researchers, they needed to be active in the research community of which they were a part. They struggled with what they wanted to do and not having the time and institutional support to do it:

I think if you had asked me in December what to do if those conferences got cancelled, I would have said, "I will finish writing those papers and submit them to a bunch of different journals, and I will pursue getting them published.”. . Finishing the paper and submitting it to a journal is going to be easy. And I will tell you that when 
I got those emails that those conferences were canceled, I don't think I have opened those documents again since then. All the other work that has come up to sort of fill this time ... that I could use for writing, and stuff just flowed in and filled that up before I even had a chance to, like, protect it. I FG09-09, RESEARCH ORGANIZATION, RESEARCHER

Those working in employment settings outside of academia also considered their sense of responsibility as researchers to introduce evidence-based practices and study major changes likely in education post-COVID-19:

We're in the middle of a curriculum revision cycle. ... So, we were really excited about embarking on this work and really diving in and taking a good look and seeing what's the best way to move forward and use some of those research-based best practices and really build something profound and rigorous, meaningful, et cetera.... [W]e're feeling like this would also be a great opportunity to really start investigating some of those best practices and looking at this as a prime time to really go after some of those goals because school is not going to be like what it was before. I FG09-05, k-12 SCHOoL DISTRICT, RESEARCHER

Within academic settings, additional dimensions of productivity were discussed, specifically about scholar activism and the value of some forms of scholarship over others. This topic raised perennial questions about implicit status hierarchies and what is valued as scholarship.

And it's pretty clear that that's what it is. It's about your scholarship, your publications, and there doesn't seem to be much room for, you know, scholar activism and-because I think that that almost disqualifies your scholarship, in a way. So, for me, the works that I work with, the spaces that I work in intellectually call for, you know, collaboration practice partnerships-working in community....

I don't study, like ... learning sciences, right? That's not necessarily a part of what I do, and so somehow learning sciences work is deemed more worthy than the work that I have. And that's not that someone has ever said that to me, but I see how they engage with that and with each other, and who they take seriously amongst each other as faculty, not just at our institution but at other institutions. I FG10-21, VERY HIGH RESEARCH ACTIVITY UNIVERSITY, DOCTORAL STUDENT

Some scholars of color also shared that they had experienced an increased volume of calls and pulls to do additional work around diversity, equity, and inclusion based on their areas of expertise, and in response to the recognizable inequities exposed by the pandemic and in the wake of George Floyd's public murder and ensuing national protests. While institutions appreciated them for their expertise, this work often took the form of service and professional development outreach rather than research scholarship, which they worried might ultimately have a negative impact on the tenure process. 


\section{Impact on Teaching: The Need to Be Inventive, Inclusive, and Intentional}

Focus group participants teaching in higher education settings reflected on the implications of the COVID-19 pandemic in the near term and for the future. They described complex conditions in their departments and programs which impacted their teaching and mentoring roles, noting that COVID-19 added extra layers of responsibility and uncertainty to their teaching. Some participants worried about the future of teaching and learning in formats adapted in their colleges and universities since the pandemic struck in March 2020. As one participant summed up:

If we are online in the fall, it's still not what I would consider virtual education. I still think of it as emergency because all of our students elected a face-to-face institution, and now they're being pressed into an online one they didn't choose. And the consequences of COVID aren't going away by fall. There's still going to be grief. There's still going to be illness. There's still going to be a loss of homes, a loss of resources for all of our kids. I FG03-41, HIGHER EDUCATION INSTITUTION, FACULTY

When asked to talk more specifically about how the pandemic had affected their teaching, many participants shared that moving classes to online platforms was not necessarily a difficult task. In fact, some participants mentioned that they were teaching online classes and that institutions had already moved a great deal of teaching to online platforms, such as Zoom. Other participants described how the shift to remote teaching and learning had been notably smooth and provided them with opportunities to rethink their pedagogical approaches to find new ways of engaging with their students. Some faculty and other instructors described their efforts as "surprisingly successful" because they, and their students, had found new ways of learning together using various applications, and of thinking more deeply, that made the enormous amount of time invested in planning and moving to online instruction worthwhile.

It is also important to note that, while some participants pointed out the opportunities, others raised issues about the inequities associated with accessing technology and fully participating in online instruction, particularly for students of color, and about whether pedagogical practices were indeed conducive to students' learning. Others worried about whether faculty and other instructors, and particularly their students, were well situated to teach or to learn from home sites, with complexities including household demands, the adequacy of private space, and sufficient equipment and bandwidth.

Many participants were stressed and described the challenges to teaching and mentoring in a COVID-19 environment. Some reflected on the huge amount of effort that was needed to restructure and change a class previously taught in a face-to-face setting into an online version in a compelling way; others thought that classes were far too long without reinvention; and still others were not sure what faculty and students were expected to achieve (for example, with respect to grading and assessment policies), generating confusion on the part of faculty and students. Participants more conversant with online learning and diverse technologies reported additional labor in being sources of support:

There were weeks of helping other faculty. Again, I teach in a fully online program. So, my curriculum and our adjuncts were okay in terms of not having to pivot our instruction, but I was pitching in across [the university], working with faculty, helping 
them-helping individuals rethink how their courses could go. So, there was a lot of extra work that happens.... So, you're willing and able to participate. I FG01-32, VERY HIGH RESEARCH ACTIVITY UNIVERSITY, CLINICAL FACULTY

One key struggle was participants' concern for both the well-being and the professional development of their students. They described interactions with their students in personal, deeply empathetic, and heartfelt ways:

Oh, yeah. I gave my students my personal cell phone number and my home number and my personal email address in case they wanted to talk. Few of them took me up on it, but I could not ignore the human side of what was going on with them. I FGo5-54, HIGHER EDUCATION INSTITUTION, ADJUNCT

Participants also struggled with the inequities their students faced in terms of remote learning. They emphasized the adverse impact on some of their students, particularly those who did not have access to reliable internet and technological resources:

I mean, my students are primarily in small rural parts of the state, and they have a different set of needs. And many of my students do not have internet or had to drive to campus to sit in the parking lot in order to attend class, and so, if you have kids, that means you're in a car with your kids having to try to participate in a class, which is for me insane. I could not concentrate with my toddler, if not a baby, in the backseat. So, in a lot of ways, I tried to support my students as best as possible, and they all did pretty well. They're very resilient, but their learning environments and situations, I think, definitely impacted them academically and emotionally and mentally throughout. | FG05-57, HIGHER EDUCATION INSTITUTION, FACULTY

Although participants primarily focused on students, in a few instances they also talked about the need for institutions not to assume that all faculty have access to internet, printers, scanners, and computers. Some faculty mentioned that they had to share their own equipment with their kids who were being taught remotely or with partners or spouses who also needed access to those resources at home.

Participants' concerns about inequities transcended access to technology, to include other dimensions of the shift to remote teaching and learning, such as how to engender equitable participation online, and what the implications were for critical perspectives and research methods.

What do critical, online, digital, pedagogical practices look like? What does just moving a course online [look like]? And so, I would hope that institutions are inviting research and discussions that are much more complex and critical in terms of understanding, well, if this is our new normal, how can this be equitable? FG02-39, VERY HIGH RESEARCH

ACTIVITY UNIVERSITY, FACULTY 
For scholars of color, the concerns revolved not only around the difficulties of teaching online, but also, in at least one case, around teaching courses on critical race theory or racism, particularly after the killing of George Floyd and the patterned police brutality. This scholar raised the concern that their university leadership did not understand what it really takes to teach these types of courses. Such courses taught online or remotely are very difficult to teach, particularly in the context of predominantly White institutions.

[We've known,] but not all of us suddenly realized how hard it is to teach anything online, let alone anything related to race or ethnicity, right? Like, all of a sudden, you've realized things that people with disabilities and special needs have always needed, and all of a sudden, now we know, right? People [are] being upset that they're being asked to start preparing to teach in the fall in person, right, and people are like, "Well, wait a minute. I don't really feel safe about doing that," I'm like, "I feel unsafe every time I go and teach," right? It might not be my physical health, right, but my emotional health, every time you expect me to go into a predominantly White classroom and talk about race and ethnicity. And they're being resistant to that, right? And so, all of a sudden, I think what this exposed is how incredibly inhumane these spaces are and how they need to be completely restructured, right? And I think that there's a range of that. I FG12-18, VERY HIGH RESEARCH ACTIVITY UNIVERSITY, FACULTY

Focus group discussions with those engaged in teaching, whether in tenure track or adjunct positions, revealed how faculty had adjusted to conditions on the ground, embraced available technology to do the best they could, and at the same time struggled with inequities and the stresses and strains of their students.

\section{Balancing Acts: Negotiating Family, Home, Community, and Professional Life}

Early career scholars and doctoral students situated their tensions, worries, disruptions, and balancing acts within the context of the larger lives they were leading. Focus group discussions powerfully revealed that multiple factors affected how participants were challenged, beyond dislocations in where they worked, their research or writing agendas, or the press of teaching or other professional commitments. ${ }^{12}$

My husband is an essential health worker. ... I was actually tenured ... two weeks before the shutdown happened... but my funding has been rescinded . . . I can't do a study that was going to cost thousands of dollars... [ [ am] really grateful for some of these opportunities ... but at the same time trying to balance classes, grading, research by myself with a two- and three-year-old, while having to worry whether COVID is coming home to us and things like that. It's been very overwhelming. I FG03-44, HIGHER EDUCATION INSTITUTION, FACULTY

\footnotetext{
12 Specifics of situations varied but complexities abounded. Approximately $30 \%$ of focus group participants were parents, with a large number noting infants and toddlers; several others reported that their spouses or partners were in health-related professions that put them at risk; several indicated that they were pregnant; and several were addressing cancer diagnoses.
} 
Moving work into the home caused disruption and challenges emphasized by many. Focus group participants discussed having difficulty in setting up work spaces, finding privacy for work, and having the right kind of equipment to work remotely. Some participants talked about having to work in cramped quarters with spouses, partners, or other household members working from home; others anchored on juggling the needs of children who also were at home. Some also talked about their struggle to create separation between work and home now that both were in the same place. Participants reported that it could be a challenge to find the mental focus to work when at home with many demands; it could also be a challenge to create boundaries and turn off work and step away from it.

And then I'm also wondering how-because I know this has changed for me, the lack of certainty and structure that I had before is really creating these long and elongated workdays, and so, like, how people's work hours have shifted, I think, matters because there's a lot of - I call it "fake working." Like, I am sitting at my desk. I'm trying to think, but my mind is everywhere else, and, you know, the other day, I was at my computer almost from nine to nine. I would have never done that under normal circumstances, but I had to get things done. But my brain was everywhere. I FG10-21, VERY HIGH RESEARCH ACTIVITY UNIVERSITY, DOCTORAL STUDENT

Although working from home presented serious challenges, a large part of participants' dislocation related broadly to the COVID-19 pandemic, the erosion of any sense of normalcy, and worries related to family and community, including colleagues and students. Substantial energy and time were put into supporting families and helping communities deal with the disruption and trauma caused by COVID-19. Participants were concerned about their families' physical and mental health, and some worried about not being able to be present to help them go through this pandemic. For some participants, parents were a source of concern and emotional labor, as they tried to arrange care from a distance, or simply were worried about the health and safety of family far away.

I think during COVID, just finding ways to be supportive of my family, like ordering groceries, that type of stuff, when I couldn't be there, making sure that family understood that they needed to stay home and what could I do to make sure they stayed home. I FG12-17, VERY HIGH RESEARCH ACTIVITY UNIVERSITY, FACULTY

The types of challenges and care needs in families were expansive and pressing. Some participants had moved for graduate school or faculty positions, placing them far from the communities in which their families lived. Many were feeling that distance particularly acutely during the pandemic. Some had taken the step of leaving their current homes to be closer to their families; others were connecting with family needs from afar.

In terms of helping out more, I mean, like, my brother ... it's hard to get ... academic support ... for family. So, you know, l've had to kind of step up a little more, and ... I'm not there, so it can get a little complicated, you know, since we're doing it through ... Google Hangouts, like, trying to figure out his math homework, you know, and so that's definitely ... it's been hard on my family and then hard on me trying to be there 
and so, you know, also just helping family try to understand everything that's going on, ... I just try to help my family ... process everything that's going on too. I FG10-24, VERY HIGH RESEARCH ACTIVITY UNIVERSITY, DOCTORAL STUDENT

Mom lost one of her jobs due to the pandemic, wife's parents both lost their jobs. The little money [we] make as grad students, [we're] thinking about how to support [our] parents. Makes it more complicated. No children, but with parents there are people [we] feel responsible for. I FG06-12, VERY HIGH RESEARCH ACTIVITY UNIVERSITY, DOCTORAL STUDENT

Many participants reflected on being grateful and privileged in comparison to others with far less in society.

So, I can say that it's been a lot. I can say that I've tried to be as positive as possible because amidst all of the negative things that I've shared, I'm incredibly grateful. I have my health. My kids are safe, my extended family as well. I FG11-04, HIGHER EDUCATION INSTITUTION, CLINICAL FACULTY

Nevertheless, participants also noted that they faced uncertainties (including uncertainties about financial and longer term economic and professional situations) and barriers in relation to research productivity while juggling other home- and family-based challenges (raising young kids, being a teacher to older kids, taking care of family members, teaching, and so forth).

For many participants, having children at home changed everything..$^{13}$ Participants with children, and young children in particular, talked about the ways that being home with their children affected multiple aspects of their working lives. They reported having limited time or bandwidth left for work after attending to their children's needs, including taking on teaching roles. They also talked about how schooling from home exerted a tremendous pressure that caused frustration and exhaustion, leaving them little room or capacity to focus on their own professional work.

Participants recounted that they were often interrupted by their children while they were trying to engage in some work activities.

I would say l've noticed a distinct difference in ability to be productive in terms of scholarship, ... when I write, I get into a groove, right? Like, I sit down. It starts coming, and I just have to get it out. So, I just keep going, going, going, but I can't do that right now because-I swear-every two seconds, I'm hearing from the other room, "Mommy, come here. Mommy, come help me." Everything is disrupted. So once that happens, I have to get myself back to where I can be productive, and then again, the same thing. Somebody is hungry. Somebody can't do their assignment. Somebody can't find their shoe. And so, I feel like my production in terms of the quality and ability to get some work accomplished definitely has fallen back, whereas some of my colleagues, l've noticed, do not have that problem. I FG03-41, HIGHER EDUCATION INSTITUTION, FACULTY

13 Note that our obtained sample included proportionally more women than men and purposefully more people of color than in the population from which they were drawn. 
Further, participants also talked about both the impossibility of providing full-time childcare and working, and about the gendered dimensions of this disruption.

Unless we ... solve the childcare, it is impossible. There is no more than 20 hours in a day, and I have to expend at least, like, 18 hours of that in childcare, so-and I have to sleep at some point. So, there is no way I can do more than this. It's impossible. So, I think, you know, I understand that it is very hard for everyone in every way. There's no race to who has it harder, but it is just impossible for parents. So, unless we get there and do something for that, it's not going to happen. Nothing happens for us. We're going to be the lowest of loW. I FG04-48, VERY HIGH RESEARCH ACTIVITY UNIVERSITY, RESEARCHER

I'm in my third year on the tenure track at a research institution and had a baby last year, so haven't taken a tenure clock extension yet for maternity leave, but I have a two-yearold and a 13-month-old that have been home with me for over 75 days. And so, my time has been incredibly reshaped. ... I'm so grateful to have somebody else here with me to co-parent, but his work has been-is-he's working from home but has an inflexible schedule, and he also has-is under threat of furlough. So, his, like, his co-parenting during the week is-like, he needs to be working, like, overtime to be-like, because he's worried about losing his job. So, l've been trying to do the work of teaching, research, and service at night and on weekends, and working very long days and supporting students and colleagues who are also in similar places. I FG04-45, VERY HIGH RESEARCH ACTIVITY UNIVERSITY, FACULTY

The realities of the COVID-19 pandemic and the ensuing alteration of social institutions like family, work, and school create conditions that can make research and academic careers exceedingly difficult. Such situations also exacerbate inequities that could have lasting effects on future generations of education researchers and the production of education research.

\section{(4) The Emergence of a Dual Pandemic and Confronting Racism}

From the onset of COVID-19, there was wide awareness of the disproportionate impact of this pandemic on communities of color. In the midst of COVID-19, a second pandemic after the killing of George Floyd heightened participants' existing concerns about the prevalence of systemic racism, anti-Blackness, and institutionalized inequities in society.

Early career participants of color reported being stressed and stretched thin by multiple demands. They also observed that higher education institutions and research fields were not themselves impervious to being sites of racism, systemic bias, and microaggressions. In the wake of the second pandemic, these participants shared that scholars of color had been asked to do even more professional work. Such expectations included supporting students of color in a department where the participant was the only Black female faculty member, teaching courses about race, or being the "go-to" person when racism and police brutality became more visible: 
My chair sent out an email today that named-well, named Black-violence against

Black people but then also said, like, "I care about people of color in the communities of color," which was a change, because it is a very White homogenous department. But, at the same time, there has been an increased request for training, or "Now what do we do?" and "Can you share your resources?" and then can potentially we make that into a certificate program. And it's just, like, a whole bunch of stuff that is-has made me just feel really overwhelmed....

And so, I worry about the fact that they-before, the university did not understand intersectionality. I am not sure they understand it now, but there is an increased ask of me to do more. ... I am just asking for, like, acknowledgment of how hard this is ...

not to be able to divest in any way ... because we teach it, we live it, we research it. So that's sort of where I am. I FG12-19, VERY HIGH RESEARCH ACTIVITY UNIVERSITY, FACULTY

In our focus group discussions, faculty of color expressed concerns that other faculty and administrators did not understand who they were and the strengths, wealth, and assets that they brought to their institutions. They felt that key institutional actors failed to acknowledge and value the "labor of love" that was required to support equity in their institutions and mentor students of color.

And to me... we like to think about the pipeline in terms of research, right? The pipeline in terms of bringing people into the tenure track, but we're also not thinking about the pipeline of . . I I don't know what to call it. The pipeline of, like, pain, right? We've chosen this path, and we tell you to come. And we help you, and we encourage you to get there. And then we put you into that pipeline, where we have not done the work to help you just be-be a scholar of color, let alone to try to thrive, right? ... [I]t's not about the individual. It's not that the individual doesn't have the skill set, right? It's not about just giving a job-let's give you a job, and then you prove that you can do it. Why? Because the institutions need to change, right? ... Like we cannot start this conversation without an acknowledgment of why you've hired us ... and what you need to do in order to ... facilitate that others engage in this work. And it's not just you. It's not the leadership. It has to be across the board, right? I FG12-18, VERY HIGH RESEARCH ACTIVITY UNIVERSITY, FACULTY

Scholars of color variously indicated that they felt numb, exhausted, and frustrated and wondered how to achieve genuine change in academia and other research settings.

Now transitioning to when the murder of George Floyd occurred, it hit me in a way-like, of course, there was Arbery, Ahmaud. That occurred initially, right? And these things have become so frequently, have become so frequent, that it's like, "Okay. Just another one." But the death of George Floyd just-I don't know. For me, it broke-it broke my spirit a bit, a bit more so and then you cry.

And in terms of the institution, my department chair-l'm fortunate to be in aexcuse me-in a department that has people of color. I'm not the only Black woman 
in my department. I value it. I'm thankful for that because I get guidance from those individuals. However, the people in leadership, the people up top in terms of the university admin, they're all White, and an email was sent out in response to, you know, the racial tension that's occurring. And some of our students felt that it was a bit tone deaf. That's typically, you know, sometimes how it goes. So, we've tried to build community in terms of our department and having Zoom meetings with students, giving them time and space.... | FG11-02, HIGHER EDUCATION INSTITUTION, FACULTY

Faculty of color were also handling increased emotional distress experienced by their White colleagues who were trying to make meaning of the protests and of what their own roles and responsibilities were.

What was interesting is that, before, no one would really reach out to say-or to ask, "Hey, like, I heard that another, like, Black person was killed. Can I help?" There was something about this time that there are a few people that have reached out in my department via text message to say, "I don't know what to say, and I don't know what to do. But I just wanted to let you know that, like, I'm thinking about you and your family. If I can help you in the work that you do in any way, please let me know, but I don't know what to do." So I was very thankful for that because that was different from-l've been here for four years, and that's been very different than any other message | received. I FG12-19, VERY HIGH RESEARCH ACTIVITY UNIVERSITY, FACULTY

Early career scholars acknowledged the challenge and the necessity of addressing systemic racism in their institutions. While some were deeply troubled by recent institutional responses, others-while also troubled-saw indicators of change that they had not observed before. These findings suggest that there is still much work to do to ensure that research and academic institutions engage in meaningful policy, climate, and culture change.

\section{Employment Trajectories, Uncertainties, and Deferments}

Focus group participants expressed heightened concerns about their employment status and career trajectories. Even while participants were in the midst of addressing immediate needs and readjusting their lives (including for some, where they lived), they were planning for the job market and grappling with present or future employment possibilities.

Participants' expressions ranged from serious concerns about the year ahead to recognition that their jobs or support packages depended on their work status, type of employment, or doctoral student status prior to COVID-19. Those on a tenure track but not yet tenured focused on how and when they would navigate promotion and tenure. Other participants expressed uncertainly or anxiety about their work status; work contractual arrangements; and institutional responses to retention, recruitment, and promotion opportunities.

Some comments reflected the tenuous context of contingent faculty and those in non-tenure track positions that predated COVID-19. Nevertheless, the pandemic exacerbated the layers of uncertainty that have flowed from budget cuts, reduced enrollment, and elimination of programs. Participants reported that institutional responses were mixed and sometimes confusing, which added to their stress 
as recruitment was frozen or layoffs announced. Others reported on more precarious situations as their contracts were uncertain or they learned that they were not being renewed. Any number of participants recognized and appreciated that institutions were sometimes also grappling with substantial uncertainty, and they valued transparency and support when it occurred.

Participants with more stable employment conditions (e.g., longer term funding or tenure) sometimes felt "privileged" to have job security. In some cases, they said they felt guilty that they were secure while many others in their professional cohorts were so adversely affected. In contrast, as a result of COVID-19, many scholars, particularly those in temporary positions at their institution (i.e., contingent and nontenure track), were notified that their contracts would still be renewed but only for a short period of time, or were waiting to hear if they would obtain enough grants to continue being employed.

I had a meeting with our dean.... And she advised [me] and my colleague, who is also another clinical professor, that she was extending our contract, yes, because our contracts are up in August. But instead of the two-year contract that we get, it was a one-year extension, and it was a terminal contract. So that this next coming year would be my last year at the institution amidst COVID. I FG11-04, HIGHER EDUCATION INSTITUTION, CLINICAL FACULTY

I am in a research position, research professor position, which means that my salary is dependent on the grants that we have. And anytime that those grants stopped, I am also stopped, ... I am in a lot of anxiety about what comes ahead, but at the same time, I'm just doing whatever research is, that is part of our job. I FG04-48, VERY HIGH RESEARCH ACTIVITY UNIVERSITY, RESEARCHER

A similar range of situations and levels of anxiousness were evident among those education researchers working in settings outside of higher education. For example, one participant in a STEM research position in a membership association worried about the economic impact of COVID-19 on their association and how the current circumstances would affect research plans (FG07-27, professional association, research manager). Another researcher in a higher education association was anxious about the precariousness of their position, which relied on research grants and soft funding and poignantly made the point, you "don't learn this in a Ph.D. program" (FG07-25, research organization, researcher).

Doctoral students also varied by where they stood in their degree programs. Some had secured academic or postdoctoral appointments or positions in research organizations or other non-university settings and were looking forward to their new posts and completing their degrees. Doctoral students who had planned to finish their programs in a year or so, discussed very explicitly that they were waiting to see what the job market would look like to decide whether to defer their graduation.

One of the conversations that I have been having with mentors just in the last couple weeks is about "Am I going to try to potentially extend my time in the doctoral program for another year, so as not to graduate into what is bound to be, like, the worst, you know, job market ever?" FG08-31, HIGH RESEARCH ACTIVITY UNIVERSITY, DOCTORAL STUDENT 
Doctoral students also mentioned that they needed to learn more about how to pivot their occupational trajectories, since many doctoral programs did not prepare them for various career paths and for adapting to an uncertain labor market. Several participants expressed the view that they were not sure whether they would be able to stay in academia-a decision directly attributed to the effects of COVID-19. One participant, with a spouse also employed at the same university, said:

It's very frustrating to the point where l've actually started looking at alternative careers outside of the academy, which is something that I don't want. Ideally, I want to stay in the academy and do the scholarship that I feel is important to do, but at this point, I don't know if I have a choice. And that's where I am right now. I FG05-58, HIGHER EDUCATION INSTITUTION, RESEARCH ADMINISTRATOR

In addition to these concerns, some doctoral students were facing significant financial challenges due to COVID-19. Others expressed feeling privileged being in academia with secure support packages and described their current status as "being in a bubble" that had, to some degree, protected them from many of the impacts of COVID-19. However, many still reported feelings of tenuousness as summer teaching opportunities were precluded or they contemplated uncertainties regarding funding for future years beyond their secure support.

Participants reflected various levels of resilience, concern, or anxiousness about financial insecurity ensuing from impacts in the economy due to the COVID-19 pandemic. Participants with stable household income or funding were grateful for their financial security and good health. One student captured this view:

I could start by saying that prior to three weeks ago, I was feeling, like, privileged, like a lot, throughout the entire COVID situation. I have a roof over my head. I had food at my table. I was able to keep my job as a graduate student. I didn't have to worry about funding. My family was healthy and in the same-their situation. They had roofs over their heads. I FG10-22, VERY HIGH RESEARCH ACTIVITY UNIVERSITY, DOCTORAL STUDENT

Others framed financial impacts in terms of future capacity for educating family, capacity to afford rent or mortgage, health insurance, and so forth:

I currently am the bulk of the income, you know, but that being said, even a little bit of a bump downward could be very disruptive in terms of just like, okay, so we have a mortgage that is out to lunch. I FG11-01, HIGHER EDUCATION INSTITUTION, FACULTY

The unknowns of the public health pandemic's trajectory challenged normative career planning for education researchers and caused significant stress and anxiousness as they looked ahead. The financial hit to higher education institutions and the shrinkage in enrollments in higher education, they recognized, could drastically affect the work sector where they sought and hoped to lead their professional lives. 


\section{Institutional (In)Capacity to Respond and Support}

Early career scholars were working at a range of higher education institutions, including public and private universities, liberal arts colleges, and community colleges. Those outside of academia were employed by research organizations, scientific societies, and K-12 schools and school districts. Combined, they were located in more than two dozen states. Doctoral students were pursuing degree programs in universities with either very high research activity or high research activity across every region of the country. All of these institutions varied in resources, climate, and culture independent of COVID-19, and were potentially experiencing different economic impacts as a function of the pandemic.

Focus group participants saw and acknowledged these differences:

But I know it seems like all of us come from, like, very different types of programs or programs that ... I know from my program, a lot of the students are working professionals and are doing this, taking classes in the evening while they worked during the day. And I think that I'm really glad that, like, all of our perspectives are here because.... it kind of shows that we're all in different places and how institutions [that] are supporting us are much different based on how-you know, what the institution is like. I FG08-30, HIGH RESEARCH ACTIVITY UNIVERSITY, DOCTORAL STUDENT

So I feel supported, and I have to be honest. With that, with the course offerings, I think that institutions, at least the two that I work with, have moved quickly. They produced resources for professors on how to quickly move your courses online, how to infuse humanistic approach to teaching a language. I FG01-34, VERY HIGH RESEARCH ACTIVITY UNIVERSITY, ALSO AFFILIATED WITH HIGHER EDUCATION INSTITUTION, FACULTY

Some focus group participants working outside of academia in organizations or associations were "not sure what [these institutions] could communicate at this time," and "thought they were doing the best they can" (FG07-27, professional association, research manager). Others expressed doubts, noting that the responses from their institutions were "passing, but not sure they are recognizing other people's anxiety" (FG07-26, high school teacher).

While some early career scholars at higher education institutions and doctoral students appreciated what they viewed as sincere efforts by their institutions to support them, few saw the leadership in their institutions as attuned to their situations; others experienced the responses as technical and bureaucratic. A number of doctoral students commented positively on being briefed by their institutions on benefits and support related to COVID-19.

Some faculty members or those otherwise engaged in teaching expressed a sense of support from their institutions in the transition from place-based to online courses:

Our dean is very transparent as well, and the communication has been very constant, very transparent, very clear. And one thing I have felt very supported in is... 
transitioning my classes to fully online. The university's response was very-I would say very supportive, and I felt that that was a good resource for me to transition my classes during a stressful time. I FG02-35, VERY HIGH RESEARCH ACTIVITY UNIVERSITY, FACULTY

Others reported that institutional messages missed the mark, and the focus on budgets, enrollments, and hiring freezes felt dehumanizing and bureaucratic.

So I think, like everybody else, the university is definitely more focused on students, student enrollment, and the money that kind of evolves around that and less so about faculty research at this point. Although just today at a meeting, there was ... finally for the first time... kind of a lively discussion [when] an administrator made mention that there might not be funds for the in-house faculty research grants that are usually offered. So I don't know if that was just a heat-of-the-moment statement or if it's true. So that's kind of where we stand. I FG03-40, HIGHER EDUCATION INSTITUTION, FACULTY

At times, these messages seemed misaligned with the emotional and human demands of the situation for early career scholars. Paradoxically, it appeared at times that institutional communications were meant to perpetuate a sense of normalcy, even when the content and circumstance were anything but normal. This paradox seemed, to focus group participants, to obfuscate the desire for relational understanding of their concerns and struggles.

... eventually, I think they sent out one of those, you know, "We really appreciate what you've been doing now," like, from the dean. So that was something, but I think it came so much after, almost like the business-as-usual kind of messaging. ... I've learned [about] myself, if I didn't know it before, that I'm not necessarily a business-as-usual person. It's not business as usual. We need to say something is happening here, and we need to give our people some space to acknowledge that and to know that we are acknowledging that. So I think that this-the managerial part-has taken over way too much of the recognizing that even part of the managerial is understanding these are humans that you're working with and that that's part of the job that you're doing. I FG11-03, HIGHER EDUCATION

INSTITUTION, FACULTY

Where scholars saw a need for relational leadership that operated on a more human scale, institutions seemed to pivot operations by holding additional meetings on online platforms.

In some ways, it's been stressful because the approach that has been taken for the most part in my department has been kind of, well, just, you know, act like everything is normal and we'll be good, and I'm just like that's not how I operate. I don't want to have 50,000 Zoom meetings for nothing. I don't need another meeting on my schedule, just that I have structure. l'll find something else to do, is kind of where l'm at, ... it's been like a tone deafness around, well, what are our students dealing with? Because we're an institution 
that has primarily first gen, students of color, students who are transfer, nontraditional students age-wise as well. So it's just like-to me, it's just like common sense would say, ask how are things different right now, but that hasn't happened. I FG11-01, HIGHER EDUCATION INSTITUTION, FACULTY

Overall, participants who spoke to the issue felt that institutional leaders had missed the opportunity to model the importance of relationships during times of crisis. Some scholars in our sample found attention to relational issues to be imperative for their own teaching and learning activity and would have appreciated more of such expressions in the leadership of their administrations.

Participants also focused on institutional response and support in the context of discussing the impact of COVID-19 on tenure and promotion. While a handful of faculty reported that their promotion schedules had not changed, one concrete policy response that seemed common across research universities was to establish processes for extensions to the tenure clock for tenure-track faculty members. Generally, faculty reported feeling conflicted and overwhelmed about a potential clock extension. They recognized the future potential for different interpretations and possible stigma associated with taking what might be construed as extra time to go up for tenure. As an institutional response to the pandemic and supposedly in support of faculty development, the tenure clock extension alone fell short for some participants.

... in addition to, like, all of the uncertainty around the job market for promotion and tenure questions. A lot of universities-it seems like mine included-are requiring faculty to decide by the end of the academic year or beginning of the next consecutive year or by the end of the calendar year, so within the next few months. But without knowing whathow long this will last and how drastically our work will be affected, I feel like if I were going up for tenure in the next year or two, I could have a better prediction of, well, you know, do I have enough things in the pipeline? Will I be ready and so on? But in thinking about going up for tenure in four years, I have no idea what things will look like in four years, and so if this is the one-time shot that this is offered, you know, to take it or not is a huge-it's a huge question. And there are no easy answers there, right? I mean, some folks are experiencing a lot of increased productivity. Others are experiencing really decreased productivity. And so to say ... it's a complicated issue, but without knowing how long things will last, it seems unreasonable to expect us to make these huge decisions in the next couple months. I FG05-55, HIGHER EDUCATION INSTITUTION, FACULTY

As a policy response intended to support early career scholars, extending the review period for tenure was necessary and important; but pre-tenure faculty felt that it might not have been adequate. The uncertainties with respect to the potential future implications were joined by a desire for clearer expectations as well as greater flexibility.

I think our institution could have done a better job of relaying to faculty and the students what the expectation-the changes and expectations would be, given the current situation. I FG05-58, HIGHER EDUCATION INSTITUTION, RESEARCH ADMINISTRATOR 
Many participants also recognized that there were dramatic and unanticipated new demands on their institutions and that their institutions were endeavoring to respond, even as participants found the responses to be inadequate in some areas. For those in academia, professional training for online teaching seemed to be the primary area of support directed to faculty members, postdoctoral scholars, and doctoral students, which was appreciated and found to be useful by many. However, participants experienced acknowledgment of and support for personal and career concerns as largely unmet.

\section{(7) Emerging and Lost Connections, Communities, and Communication}

Focus group participants uniformly saw connections, community, and communication to be essential and to merit being cultivated during COVID-19. Participants reported that the pandemic had affected their sense of connection to colleagues and/or the support they received from colleagues. For some, creating spaces for these connections to happen (e.g., writing groups, informal gatherings) was important during this pandemic. Many discussed the impact of reduced university resources on their opportunities for professional development and on paying for memberships to professional associations, for research support, and for attending conferences. Those working in settings outside of academia similarly expressed concern that they did not have the bandwidth or resources to remain as professionally engaged.

Many participants raised issues relating to networking. Some expressed a sense of loss from not being able to attend conferences and other such activities. Others noted that they had created virtual networking opportunities. Virtual happy hours, coffee breaks, and writing groups were frequently mentioned as important. More general networking events, such as with departmental colleagues, were more mixed in terms of their usefulness to the participants.

And I think what did sustain me, initially I had a couple of colleagues that we had a time blocked out every day for writing, and that was even just like a check-in of just havingjust seeing faces every day. It was-I didn't know how important that space would be, and so that was an opportunity just to check in. Or if we didn't do it virtually, then we would text, just making sure we were okay, or - I did have deaths in the family and attending virtual funerals and not being able to be home. I FG12-17, VERY HIGH RESEARCH ACTIVITY UNIVERSITY, FACULTY

But, in some ways, I see [our] communities coming together. Certainly, our online community, our students are sharing in ways that are far more personal, and they're both faculty, our adjuncts, and our students taking the lead in conversations from mentoring groups, informal mentoring groups, working with $\mathrm{K}-12$ teachers that they know in order to be able to help them support the shift in online teaching, which is lovely. So there are some lovely things that are happening. I FG01-32, VERY HIGH RESEARCH ACTIVITY UNIVERSITY, CLINICAL FACULTY

Doctoral students, in particular, also expressed worry about the effects of diminished opportunities for connections and community on their job market prospects. They seemed particularly concerned about their chances of getting tenure track positions in a job market that was incredibly competitive 
to begin with and now was facing budget cuts and hiring freezes. They questioned their ability to create networks and to stay connected with people who might potentially be hiring in the absence of in-person meetings and conferences:

But that's just something that I think about, like-like, because I feel like when the official job market dries up, then you're supposed to hit all the alternative vectors, right? Like, you're supposed to try to network and run into people and, like, do all this stuff, and so much of that stuff is based on close contact and, like, opportunities for that contact. So, yeah, I don't know that there's a solution to that, but that's something that's definitely on my mind, too, is like how am I ever going to get in front of people with decisionmaking power now when l'm going to probably be at my computer for the duration, you KnOW. | FGO8-31, HIGH RESEARCH ACTIVITY UNIVERSITY, DOCTORAL STUDENT

The extent to which COVID-19 will impact budgets, hiring, institutional sustainability, and professional associations is yet to be fully realized or understood. Nevertheless, early career scholars could clearly foresee the likely long-term impact on research and on the workplaces where education researchers reside. 


\section{Recommendations}

O ur findings in this report are limited to this one study of 12 focus groups, 58 participants, and 18 hours of discussion and recording. In offering recommendations, we appreciate that our findings should not be overgeneralized. Nevertheless, we believe that the experiences of our focus group participants have implications for the kinds of actions that higher education institutions, scientific and scholarly associations, funders, and other leaders might contemplate, and we offer them in that spirit.

We also offer these recommendations with an awareness that we are in the midst of a severe resource shortage at universities, and we acknowledge that institutions may have taken additional steps since the focus group data were collected. Thus, our recommendations may be similar

It is our great hope that institutions, and our field more broadly, might embrace this moment in ways that create important and needed changes. or supplemental to existing efforts. It is our great hope that institutions, and our field more broadly, might embrace this moment in ways that create important and needed changes in practices and policies that contribute to equitable and enriching places for early career scholars and doctoral students to work and thrive.

\section{(1) Provide Funding That Includes More Material Support to Emerging Scholars}

This support could come in various forms, such as increases in awards with attention to salary coverage (in particular for those on "soft" support), childcare stipends for faculty and researchers with young children, health and mental health care benefits, housing, childcare, health insurance counseling, and so forth. As a consequence of COVID-19 and related research and employment disruptions, there needs to be attention to bridge funding to permit exiting and early career scholars to publish from their research and develop new studies.

\section{Refocus and Invest in Teaching and Pedagogies}

Some focus group participants saw new opportunities for research and for teaching in the post COVID-19 environments. The emphasis on technology and the introduction of advanced platforms, for example, inspired some transformative work and related research in pedagogical techniques, reconstructing learning environments, and innovations in building upon human and material resources.

\section{Build Community and Connect Scholars}

Professional societies and other higher education leadership organizations have an important role to play in crafting strategies and solutions to conditions created by COVID-19. Several focus group participants said that they joined the focus groups to network with others similarly situated, and to learn more about what organizations were doing to confront critical issues created by COVID-19 and to plan for the future. Others emphasized that professional societies could do much to promote discussions among various groups (e.g., through forums, webinars) on issues facing emerging faculty and researchers. 


\section{Involve Faculty and Early Career Researchers in the Decisions That Will Impact Their Work and Career Trajectories}

Faculty voices need to be engaged in developing explicit statements and processes around tenure review and/or contract extensions. While many institutions have already offered tenure clock extensions in response to COVID-19, institutions might consider additional measures to meet early career scholars' needs. Given the disproportionate impact that the pandemic has brought to many scholars of color in a range of contexts, and to others in time-limited or uncertain positionings (e.g., postdocs, faculty on term contracts), there should be special attention to these groups and their professional well-being. Senior faculty members should role model responsible faculty governance while also commending collective faculty voice in institutional decision-making.

\section{Support Scholars With Caregiving Responsibilities}

Acknowledge that scholars with caregiving responsibilities have additional jobs, roles, and challenges, particularly while attempting to get academic work done and to be productive in the kinds of ways that research institutions reward. The pandemic has most likely also heightened challenges for all researchers, in particular women, who care for children, elderly parents, or other persons. Our sample was such that we had the unique benefit of listening to more mother scholars of color. As has been shown across multiple spheres of public policy, when women of color, and particularly mothers, are centered in policy for social uplift and empowerment, all social groups benefit.

\section{Focus on Mentoring}

Perhaps the most direct action that institutional leaders and senior members of the education research community can take to address the concerns, issues, dilemmas, and inequalities produced by the pandemic is to reach out and offer support to early career scholars. Leaders can set time aside for discussing possible redress for extra work placed on those doing diversity trainings and those supporting the transition to remote/online learning. Leaders and senior researchers can support early career scholars in problem solving or designing pathways forward in their research and careers. Colleagues can invite participation on still-active research projects, share existing data, co-author manuscripts, and intentionally extend their professional networks.

\section{Acknowledge Racism and Inequities in Academia and Other Research Settings, and Take Anti-Racist Action}

Educational institutions (e.g., higher education settings, research associations, public and private funding agencies) can take a lead in reviewing and revising their policies to address the enduring consequences that this pandemic will have for the academic and scientific trajectories of early career education researchers, especially scholars of color. To be most powerful and relevant, policy development should avoid colorblind and gender-neutral approaches and solutions that may not target resources effectively. 


\section{Conclusion and Moving Forward}

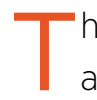
his moment presents an unprecedented opportunity to redress longstanding inequities. Whether acting interpersonally, organizationally, or systemically, institutions, leaders, and senior scholars can work to effect change in support of the next generation of education researchers. The above recommendations are merely a starting point that reflects the experiences shared with us by early career scholars and doctoral students.

Our inquiry does not end with this report. We have a major national survey of early career scholars and doctoral students in education research underway that will add to our understanding of the impact of COVID-19 on the wide and diverse population that constitutes this field. We are also working with investigators leading similar

This moment presents an unprecedented opportunity to redress longstanding inequities. studies in other fields of science and scholarship so that together we are able to construct a fuller picture of institutional practices, challenges, and changes prior to, during, and as a consequence of this dual pandemic. We plan to release a second report later in 2021 with preliminary analyses of what we have learned through the survey about the impact of COVID-19 on the lives, experiences, concerns, and perceptions of early career education researchers. We also plan to convene a forum for discussion and dialogue about this work at the AERA 2021 Virtual Annual Meeting. 


\section{Appendices}

\section{Appendix A \\ COVID-19 Focus Group Study Methodological Details}

\section{OVERVIEW}

The COVID-19 Focus Group Study is a joint project of education research scholars connected with the American Educational Research Association (AERA) and the Spencer Foundation. ${ }^{14}$ The project was reviewed and approved by the Institutional Review Board (IRB) of the American Institutes for Research (AIR) on May 8 , 2020 (Project No. EX00515). AIR serves as AERA's Institutional Review Board through a service agreement.

\section{STUDY DESIGN}

The COVID-19 Focus Group Study was designed to be inclusive of both early career scholars and doctoral students. To ensure diversity beyond the institutions at which early career scholars worked or doctoral students pursued their degrees, the 12 focus groups were designed to systematically vary aspects of years of experience and background (see Table 1).

The study design included seven groups of scholars employed in academia either in "very high research activity" universities or in other "higher education institutions," inclusive of all higher education settings (2-year colleges, 4-year colleges, and universities that were not otherwise classified as "very high research activity" universities). Within these seven groups, there were also dedicated groups of scholars of color ${ }^{15}$ and women of color, as well as early career scholars at the outset of their careers (1-4 years beyond their doctorates) and later in their careers (5-7 years out). The intersection of these design requirements can be readily seen in Table $1^{16}$

The study also included two groups of early career scholars working outside of academia.

In addition, the study included three focus groups of doctoral students: one group pursuing degrees only at "very high research activity" universities, one group pursuing degrees in "high research activity" universities, and one group of doctoral students of color in universities of "very high" or "high" research activity. The doctoral student groups included only "very high research activity" and "high research activity" universities since these are the institutions that have almost all of the programs offering research doctorates. We used the Carnegie Classification of Institutions of Higher Education to constitute these two groups. ${ }^{17}$

14 The COVID-19 Focus Group Study is part of a two-part project being undertaken by AERA and the Spencer Foundation. It was designed to be a first inquiry in its own right and also to contribute to the development of a web-based survey, the AERA-Spencer COVID-19 Impact Survey, which launched on October 29, 2020. The principal investigators of the study are Felice J. Levine (AERA) and Na'ilah Suad Nasir (Spencer Foundation).

15 Scholars of color participating in the focus group study were identified based on race/ethnicity demographic information included in the AERA membership database. Persons "of color" include those self-reporting in the database as American Indian/Native American, Asian/Pacific Islander, Black or African American, Hispanic, or a combination of two or more races/ethnicities.

16 Early career scholars employed in academia held faculty appointments or were in other positions, including postdoctoral positions, temporary instructor appointments, positions in institutional research offices or research centers, and so forth.

17 The Carnegie Classification of Institutions of Higher Education was used to identify and place participants in four focus groups of early career scholars at "very high research activity" universities. The classification was also used to constitute one focus group of doctoral students at "very high research activity" universities. In addition, the classification was used to create one dedicated focus group of doctoral students at "high research activity" universities and one focus group that included doctoral students at either "very high research activity" or "high research activity" universities. Doctoral students at institutions classified as "Doctoral/Professional Universities" in the Carnegie system were not included in the focus group study. There were also three focus groups of early career scholars who were employed at higher education institutions not classified as "very high research activity" universities; those focus groups reflected the range of other institutions that constitute higher education settings (including 2- and 4-year colleges). For further information on the Carnegie Classification, see https://carnegieclassifications.iu.edu/classification_descriptions/basic.php. 


\section{STUDY POPULATION}

The AERA 2018 and 2019 membership records served as the database for identifying persons who met the criteria for each of the 12 focus groups. ${ }^{18}$ For purposes of the study, early career was defined as within 7 years of receipt of the doctoral degree; that is, individuals who had received their doctorates from 2013 to 2019 were eligible to be invited to participate. Doctoral students were identified by reaching out to persons who were Graduate Student Members ${ }^{19}$ in 2018 or 2019 and only inviting those in doctoral programs to participate in focus groups.

Since the focus group study was directed to examining the impact of COVID-19 on those with doctorates or pursuing doctoral degrees in the United States, all potentially eligible participants needed to have U.S. mailing addresses. Also, early career scholars were eligible to participate only if we were able to confirm from the AERA database that they had received their doctorates during the criterion years. To include a person in the focus group study, we also needed to have gender and race/ethnicity data, and, for those with doctoral degrees, workplace data. Those with missing information were excluded from eligibility.

Table 1 shows by group the numbers of persons from the AERA database who met all of the focus group criteria. For each group, participants were drawn randomly from the eligible population and invited to participate. We invited participants to join groups at specified dates and times based on their attributes. ${ }^{20}$

\section{Table 1 Focus Groups by Group Attributes and Size of Population}

\begin{tabular}{|c|c|c|}
\hline Groups & Group Attributes & $N$ \\
\hline \multicolumn{3}{|c|}{ Early Career Doctorates (Total $=3,930$ ) } \\
\hline Focus Group 01 & Doctorates, Very High Research Activity Universities, 5-7 Years Out & 691 \\
\hline Focus Group 02 & Doctorates of Color, Very High Research Activity Universities & 622 \\
\hline Focus Group 03 & Doctorates, Higher Education Institutions, 5-7 Years Out & 891 \\
\hline Focus Group 04 & Doctorates, Very High Research Activity Universities, 1-4 Years Out & 752 \\
\hline Focus Group 05 & Doctorates, Higher Education Institutions, 1-4 Years Out & 868 \\
\hline Focus Group 07 & Doctorates, Employed Outside of Academia & 728 \\
\hline Focus Group 09 & Doctorates, Employed Outside of Academia & 728 \\
\hline Focus Group 11 & Doctorates of Color, Higher Education Institutions & 660 \\
\hline Focus Group 12 & Female Doctorates of Color, Very High Research Activity Universities & 442 \\
\hline \multicolumn{3}{|c|}{ Graduate Students (Total $=6,035$ ) } \\
\hline Focus Group 06 & Doctoral Students, Very High Research Activity Universities & 3,714 \\
\hline Focus Group 08 & Doctoral Students, High Research Activity Universities & 2,321 \\
\hline Focus Group 10 & $\begin{array}{l}\text { Doctoral Students of Color, Very High Research Activity Universities and High Research } \\
\text { Activity Universities }\end{array}$ & 3,092 \\
\hline
\end{tabular}

Note. Totals exclude AERA members in 2018 and 2019 for whom one or more variables of interest were missing. Individuals may be listed in multiple groups based on shared attributes but were only invited to participate in one group if drawn for the focus group sample. The abbreviated term "Doctorates" was used to designate early career scholars who had received their doctoral degrees no more than 7 years before their participation in the study. "Years Out" indicates the number of years from participants' receipt of their doctorates to their participation in the study.

18 Membership in AERA was not required for participation. The membership database combined for both years provided a resource for identifying and selecting early career scholars and doctoral students. AERA is the largest and most comprehensive education research association in the United States (with approximately 25,000 members in any one year); however, nowhere near all early career scholars or doctoral students undertaking education research are members of AERA.

19 "Graduate Student Member" is a membership classification used by almost all graduate students in AERA. Some very small number opt to be Regular Members; they were not included in the sample since they were not readily identifiable as graduate students.

20 Because groups were set a priori at specific dates and times and each group was defined by specific attributes, those interested in participating needed to be available at designated times to be included in the study. The overall size of each group may have been affected by this constraint. 


\section{FOCUS GROUPS AND OBTAINED GROUP SIZE}

Table 2 summarizes the 12 focus groups by the number of persons in each group and the groups' composition by gender and race/ethnicity. Initially we hoped to have focus groups of 8 to 10 participants (with a maximum of 12). All 12 groups were substantially smaller than the original target numbers. Groups ranged from 3 to 7 participants, with groups of 5 being modal. ${ }^{21}$ There were 58 participants in total across the 12 groups.

As we were constituting the groups, very few people declined to participate. Some did not respond to the invitational email (which included a secure portal for consent); others indicated that they would like to participate but could not do so at the designated time. Because each focus group had specified parameters, we usually could not offer an invitee an alternative time.

\section{Table 2 Summary of Focus Group Type With Number and Characteristics of Participants (Weeks of May 18 Through June 1, 2020)}

\begin{tabular}{|c|l|c|c|c|}
\hline Focus Group & \multicolumn{1}{|c|}{ Focus Group Type } & Total Persons & Females & Persons of Color \\
\hline 01 & Doctorates, Very High Research Activity Universities, 5-7 Years Out & 3 & 2 & 2 \\
\hline 02 & Doctorates of Color, Very High Research Activity Universities & 5 & 4 & 5 \\
\hline 03 & Doctorates, Higher Education Institutions, 5-7 Years Out & 5 & 5 & 1 \\
\hline 04 & Doctorates, Very High Research Activity Universities, 1-4 Years Out & 7 & 6 & 4 \\
\hline 05 & Doctorates, Higher Education Institutions, 1-4 Years Out & 7 & 7 & 5 \\
\hline 06 & Doctoral Students, Very High Research Activity Universities & 4 & 3 & 2 \\
\hline 07 & Doctorates, Employed Outside of Academia & 3 & 3 & 2 \\
\hline 08 & Doctoral Students, High Research Activity Universities & 4 & 5 & 3 \\
\hline 09 & Doctorates, Employed Outside of Academia & 6 & 4 & 5 \\
\hline 10 & Doctoral Students of Color, Very High Research Activity Universities & 5 & 5 \\
\hline 11 & and High Research Activity Universities & 4 & 4 & 5 \\
\hline 12 & Doctorates of Color, Higher Education Institutions & 5 & 5 & 5 \\
\hline
\end{tabular}

Note. The abbreviated term "Doctorates" was used to designate early career scholars who had received their doctoral degrees no more than 7 years before their participation in the study. "Years Out" indicates the number of years from participants' receipt of their doctorates to their participation in the study.

Overall, women and persons of color were much more likely to agree to participate in the focus groups. Across the 12 groups, there were 58 participants, with 86\% (50) female 22 and 69\% (40) persons of color. As a consequence, for example, Focus Group 11 became a second women of color group, although designed to be more broadly a scholars of color group at higher education institutions.

Our primary aim for the focus group study was to create opportunities for conversations about the range of professional and personal experiences, circumstances, and changes experienced by early career scholars and doctoral students in the midst of the onset of COVID-19. We created these groups using a systematic and purposeful approach in order to draw out and report on a range of early career and doctoral student voices, as well as to inform the design of the survey. Thus, we do not claim generalizability for these findings.

21 The many new time demands and life changes for this early career and doctoral student population that became evident in our efforts to constitute these focus groups were later reflected in what we learned throughout the course of the focus group study from the participants.

22 Participant genders were identified based on gender self-designations in the AERA membership database: female, male, other (with option to specify). 


\section{FOCUS GROUP IMPLEMENTATION AND DATA COLLECTION}

All focus groups were conducted virtually, using Zoom meetings during the weeks of May 18 through June 1. The groups were scheduled for 1.5 hours; generally, all operated on schedule. With the knowledge and consent of the focus group participants, all groups were video-recorded. AERA staff members conversant with the technology and delivery of virtual events administered the sessions and saved the video recordings in a data-secure location on the AERA server. Unfortunately, there were technical failures in recording two groups; consequently we have no video recordings for one doctoral student group and for one group of early career scholars employed in sectors outside of academia.

Prior to the conduct of any focus groups, a Focus Group Protocol was developed to capture the core issues of the COVID-19 Focus Group Study (see Appendix B). The aim was to create an environment to support discussion of COVID-19, its impact, and participants' experiences and perspectives, as well as cultivating conversations that would be fluid. All groups started with open-ended selfintroductions and a request that participants think about recent weeks and capture in two words their feelings about their situations in this time of pandemic. General issues as set forth in the protocol were probed, though most comments on these subjects were stimulated as a function of the cross-talk and conversation in a group.

Three research team members participated in each of the 12 focus groups. One member took the lead in facilitating the group, with a second person (co-lead) primarily raising issues that did not emerge organically in the discussion or, more rarely, asking a question. The third team member served as a dedicated notetaker. Having two leaders (lead and co-lead) facilitated keeping track of any procedures or processes that might need subsequent discussion or review.

During the course of the focus groups sessions, team members conferred and debriefed to make note of any group dynamics or overarching issues that might merit attention when the video and transcript data were examined. Also discussed at that time were any lessons learned for the conduct of the next series of groups.

\section{DATA PREPARATION}

All recordings of focus groups were transcribed and deidentified to protect explicit personal identifiable information (e.g., names, institutions) and redacted to protect deductive disclosure possibilities in conversations. Notes were also reviewed and deidentified before analysis commenced by the study team. The focus group videos were available to the study team during the analysis and writing phases through secure access in a double-secured, password-protected Dropbox. The videos also reside, as noted above, in a secure location on an AERA secure server.

\section{DATA ANALYSIS}

Identifying themes and perspectives. Data analysis for the focus group study incorporated several systematic steps to identify salient themes, issues, and perspectives. First, all team members examined the detailed notes for all 12 focus groups. Second, at least two members of the team viewed the video recordings of each group to further flesh out themes, as well as to consider how the discussions had evolved and how the points of emphasis had taken shape in the group interactions. Third, team members examined the transcripts in detail. In addition, two team members read all of the transcripts and viewed all of the video recordings as final steps in identifying themes across all 10 recorded groups. 
Based on these steps, the team identified eight salient themes as central to the focus group discussions:

1) Research Impact, Directions, and Productivity

2) Impact on Teaching and Mentoring

3) Sense of Professional Loss/Gain

4) Employment/Work Status or Doctoral Student Status (for doctoral student groups), Career Trajectories, and Financial Issues

5) Home-Family-Work Balance and Tension, Well-Being, Care, and Conflict in Competing Aims and Aspects

6) Feelings and Emotions (about a multiplicity of contexts, including positive feelings and coping skills, opportunities, demonstrations of resilience, and frustration about institutional responses)

7) Institutional Response and Responsiveness (by participants' own institutions and broadly by higher education systems, funders, and research associations)

8) Inequity/Inequality in Access, Opportunity, and Institutionalized Exclusion (including systemic racism; gender bias/identities; xenophobia; discriminatory practices; geographic, socioeconomic, digital/technological, and other divides; [dis]abilities; ageism)

Analyzing the themes and hearing the voices. The transcripts were analyzed in two steps, using the qualitative analysis software Dedoose (https://www.dedoose.com/). First, the team was divided into pairs of researchers, who examined each transcript using the Dedoose tools to identify the eight themes within the transcript. Second, each member of the team was assigned one of the themes and asked to capture in concepts, words, prototypical quotes, and summary narratives what was salient and patterned in that thematic area. These analyses were the basis of arriving at the findings by theme. ${ }^{23}$

In addition to the Dedoose analysis, the team created a merged database from the transcripts of all the focus group sessions in searchable PDF format. This data source facilitated further study and verification of the voices of focus group participants, as it allowed for searches by individual, by group, and across groups.

23 The report presents findings related to seven of the themes, using language that captures the essence of the findings for that theme. They are Research Impact: Disruptions, Delays, and Adaptations; Impact on Teaching: The Need to Be Inventive, Inclusive, and Intentional; Balancing Acts: Negotiating Family, Home, Community, and Professional Life; The Emergence of a Dual Pandemic and Confronting Racism; Employment Trajectories, Uncertainties, and Deferments; Institutional (In)Capacity to Respond and Support; and Emerging and Lost Connections, Communities, and Communication. One of the themes, Feelings and Emotions, was considered in the "Two Words to Start" section at the beginning of the findings and also gave voice to the findings in the other seven sections. 


\section{Appendix B \\ COVID-19 Focus Group Study \\ Focus Group Protocol: Guiding Issues and Questions}

Appendix B summarizes the protocol for the three components of the focus groups in the AERA-Spencer COVID-19 Focus Group Study.

\section{COMPONENT I: BRIEF INTRODUCTION (10 MINUTES)}

a. Welcome, thanks, and reminder/overview of our aims (leader introduces self). The reason we invited you to participate in this session is to better understand the impact of the COVID-19 pandemic on your research and professional trajectories. We specifically want to learn more about your current situations and experiences, how your research agendas and priorities are being shaped as new questions and issues arise as a consequence of the pandemic, how your institutions/workplaces are addressing situations like yours, and what strategies you might recommend that they or other organizations pursue to help you move forward professionally and personally. These focus groups will contribute in themselves and also inform a survey that will be conducted nationally.

b. Introduction of research team (leader introduces co-lead and notetaker). This focus group was designed to have a small group engage in reflective and meaningful conversation. As we noted in our outreach, we are video-recording and transcribing the sessions for research purposes only. Our aim is to facilitate a free-flowing discussion.

We want to remind you also that your participation is voluntary. Whether you decide to speak to an issue is entirely up to you.

Now is the time to learn about each other: It would be great if we could go around and learn about who is in the group. Please introduce yourselves and (1) share your preferred first name so we get to know each other a little bit better, and (2) use two words to describe how you all feel right nowwhich words would you use?

c. Norms, processes, Zoom logistics, etc. Along with the Zoom link, we sent logistical information and the norms to support the group. To summarize them briefly, please be sure your video camera is on, and please also mute your microphone during the session when you are not speaking. You can visually indicate by raising your hand that you would like to talk, or you can use the chat function to say "Would like a turn." You can also use the chat function if you just want to comment. Our research team will be monitoring the chat so we know who wants to participate. On the norms side, let's be sure that we are attentive to engaging everyone who wishes to talk so that, even for this brief meeting, we cultivate a mutually supportive climate.

\section{COMPONENT II: LARGEST PORTION OF CONVENING (60 MINUTES)}

a. Open-ended fluid discussion. We would like to hear from you so that we can learn what is important to focus on in a more broadly based survey. Essentially, where are you all at?

Please, whoever would like to share, first could you reflect on how the last weeks are going and what is most salient for you these days? 
b. Guiding questions. Questions on these topics may be introduced as needed to keep the conversation going and ensure some consideration of the key study issues:

- Home-family-work: new challenges, responsibilities

- Current employment/work status

- Dislocations and financial situation

- Impact on research-what and how

- How are you handling research, teaching, etc., currently?

- What do you most need as you think ahead?

- What messages are you receiving from your institution about what you should focus on or what your priorities should be?

- What are your biggest concerns, worries?

- What supports are available, and what supports are missing?

- Are you seeing or possibly experiencing any unanticipated opportunities?

\section{COMPONENT III: ROLE REVERSAL (20 MINUTES)}

If you were part of the AERA-Spencer COVID-19 research team developing the survey:

- What would you be most attentive to?

- What are some areas we might not think of that we should address?

- Are there issues of feelings and comfort levels where we might be inattentive that should be salient as we move forward?

Final thoughts? Big thank you! Contact info for us: [on screen and follow-up email] 


\section{About the Authors}

Megan Bang (Ojibwe and Italian descent) is currently serving as the senior vice president of the Spencer Foundation and is a professor of learning sciences and psychology at Northwestern University. Bang studies dynamics of culture, learning, and development with a focus on the complexities of navigating multiple meaning systems in more effective and just learning environments. She is currently focused on teaching and learning about complex socioecological systems and their intersections with power and historicity. She conducts research in formal and informal learning environments and often utilizes participatory methods with communities. Bang serves on the Board on Science Education at the National Academy of Sciences, and on the National Science Foundation's Education and Human Resources Advisory Committee and its Advisory Committee for Environmental Research and Education.

Nathan E. Bell is the director of governance and special projects at AERA. He previously served as the associate director for education research and research policy at AERA and as the director of research and policy analysis at the Council of Graduate Schools. Bell is the co-author of a number of publications about higher education and the workforce, including Pathways Through Graduate School and Into Careers; The Role and Status of the Master's Degree in STEM; Ph.D. Completion and Attrition: Policies and Practices to Promote Student Success; and The Path Forward: The Future of Graduate Education in the United States. Bell has a master's degree in public policy from The George Washington University.

Ryan Evely Gildersleeve is associate dean and professor in the Morgridge College of Education at the University of Denver. His research investigates the relationship between postsecondary education and democracy, working across the philosophical foundations of tertiary education and social research, and the cultural analysis of educational opportunity. His work takes form as philosophy, critical ethnography, and critical policy studies. Gildersleeve's current empirical research investigates the role of income-share agreements in postsecondary education affordability. His philosophical work focuses on the future of the university and a new social contract for higher education. In 2019, he was a senior visiting fellow at the Spencer Foundation. Gildersleeve received his Ph.D. from the University of California, Los Angeles. He is a graduate of Occidental College.

Matthew A. Holsapple is the managing director of organizational learning and impact at iMentor, which works with high schools to connect students with mentors, harnessing the power of personal relationships to support students toward postsecondary access, transition, and success. Previously, Holsapple served as an associate program officer at the Spencer Foundation, where, among other things, he led the grant program supporting research-practice partnerships in education. He also spent time at the University of Chicago's Consortium on School Research, leading research projects on college readiness and success for students graduating from Chicago high schools and using research evidence to help high school principals, counselors, and district leaders better understand student outcomes and develop data-engaged strategies to better serve students. Holsapple earned a Ph.D. in higher education from the University of Michigan and teaches courses on higher education policy at Northwestern University. 
Felice J. Levine is executive director of the American Educational Research Association. Her areas of expertise include science policy, research ethics, data access/sharing, the academic and scientific workforce, and higher education. She is currently collaborating on an education data hub to support discovery, collaboration, and capacity building in STEM research. She chairs the board of the Council of Professional Associations on Federal Statistics, co-chairs the Societies Consortium on Sexual Harassment in STEMM, and is on the board of the Consortium of Social Science Associations and the advisory committee of the Humanities Indicators Project. Levine is a past president of the Law and Society Association and the World Education Research Association and is convenor of the Americas Education Research Network.

Na'ilah Suad Nasir is the president of the Spencer Foundation, which funds education research nationally. She has held faculty appointments at Stanford University and at the University of California, Berkeley, where she also served as the vice chancellor for equity and inclusion. Her scholarship focuses on the cultural and racialized nature of learning. Nasir is a member of the National Academy of Education and is the president-elect of AERA. She chairs the board of the National Equity Project, serves as an advisory board member for the Public Policy Institute of California, and is on the board of the National Public Education Support Fund.

Cecilia Rios-Aguilar is a professor of education and associate dean of equity, diversity, and inclusion at the University of California, Los Angeles, School of Education and Information Studies. She also serves as a faculty co-director of Policy Analysis for California Education and as a board member of the Spencer Foundation. Her research is multidisciplinary and uses a variety of asset-based conceptual frameworks and statistical approaches to study the educational and occupational trajectories of marginalized students. Currently, Rios-Aguilar is examining how community college students make decisions about majors, jobs, and careers. Rios-Aguilar obtained her Ph.D. in education theory and policy and her M.S. in educational administration from the University of Rochester, and her B.A. in economics from the Instituto Tecnológico Autónomo de México.

Katherine J. Rosich is a senior policy analyst at AERA with extensive experience in research on a wide range of issues and projects. As a long-term consultant to AERA, she has been engaged in several major research initiatives, including on data sharing, open science, and doctoral training in education research. She participated in the development of the World Education Research Association and the Americas Education Research Network. She has also served as a consultant to the American Sociological Association and the Urban Institute, and was a senior researcher at the United States Sentencing Commission, the Administrative Office of the U.S. Courts, and the Canada Department of Justice. 